\title{
Urinary incontinence
}

\author{
D Ames, IR Hastie
}

'To accept incontinence in the elderly, in the absence of dementia, as a natural outcome of growing old is a step backward into nineteenth century medicine'.

SL STANTON

The International Continence Society defines incontinence as a condition in which involuntary loss of urine is a social or hygienic problem and is objectively demonstrable. ${ }^{2}$ Urinary incontinence is common. In a recent MORI poll of adults living at home in the UK, $16.8 \%$ of women and $13.3 \%$ of men aged over 65 years had experienced urinary incontinence. ${ }^{3}$ The corresponding numbers for patients in institutional care are significantly higher with $19.6 \%$ and $25.6 \%$ of patients, mainly females, in residential care and long-stay care, respectively, having urinary incontinence. ${ }^{4}$

Urinary incontinence may be classified conveniently into transient and established incontinence. Continence is voluntary and is an acquired function. Transient urinary incontinence may be caused by acute illnesses, acute confusional states and impaired conscious level, drugs, metabolic disorders, depression, immobility, faecal impaction, environmental factors, alcohol, or any combination of these. Urinary incontinence may be precipitated by urinary tract infections.

The commonest causes of established urinary incontinence in elderly people are detrusor instability and genuine stress incontinence, which has a prevalence of $46 \%$ in incontinent females aged over 75 years. ${ }^{5}$ Less common causes of established urinary incontinence include sensory urge incontinence, overflow incontinence (voiding dysfunction), urethral instability, fistulae, functional, post-prostatectomy and any combination of these. Stress incontinence is a common symptom (not a diagnosis) and is frequently found with co-existing detrusor instability, urethral instability or overflow incontinence.

\section{Assessment and investigation}

A thorough history and examination is essential. The history should include specific details on the severity and duration of incontinence as well as the presence of frequency, urgency, nocturia, and any precipitating factors. A past surgical and obstetric history is essential. The type and number of any protective garments used is helpful. The physical examination should include a rectal, pelvic, and neurological examination, as well as mental state and mobility.

Clinical assessment is supplemented by urinalysis, culture, and frequency/ volume charts. These provide not only an indication of periods of incontinence to which habit-training can be directed, but an estimate of the functional bladder capacity. Blood tests assess renal function, serum glucose, and calcium. Any residual bladder volume should be measured by catheterisation or ultrasonography.

Despite careful clinical assessment an accurate clinical diagnosis is frequently difficult and the correlation of clinical features with urodyanmic investigations is poor. Algorithms have been attempted and revised. ${ }^{6,7}$ Other have found that symptoms, signs and residual volumes are helpful in diagnosing detrusor instability and voiding dysfunction, but not an incompetent urethra. ${ }^{8}$

Accurate diagnosis requires urodynamic studies, but not all incontinent elderly patients require these investigations. Opinion is divided, but doubtless all patients in whom an adequate trial of conserative management has failed, and in whom surgery is being considered, should be investigated. ${ }^{9}$ Others believe that urodynamics are more relevant in an elderly population where a poor memory and sensory deficits lead to a substandard history and in whom a specific urodynamic abnormality is likely to be found. ${ }^{5}$ Filling cystometry is the diagnostic investigation of choice. Where genuine stress incontinence and detrusor instability co-exist, urodynamic studies allow the clinician to decide where to target the initial treatment. Voiding cystometry is helpful in the diagnosis of outflow obstruction which may co-exist with other causes of urinary incontinence. Complex cases may require synchronous video-cysto-

Division of Geriatric Medicine, St George's Hospital Medical School, London SW17 ORE, UK

D Ames

IR Hastie

Correspondence to IR Hastie

Accepted 8 June 1994 


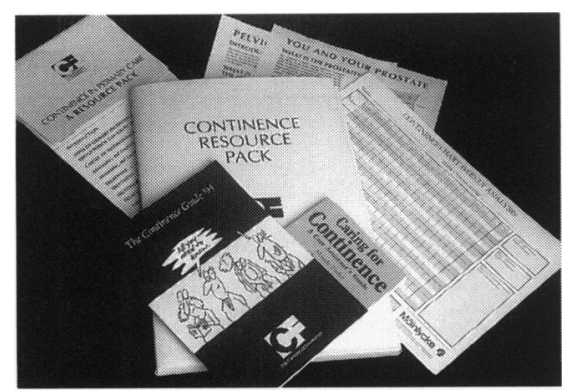

Figure 1 A wide selection of literature and advice, for all agencies, is available from The Continence Foundation, 2 Doughty Street, London WC1 2PH, UK (tel: 0171404-6875)

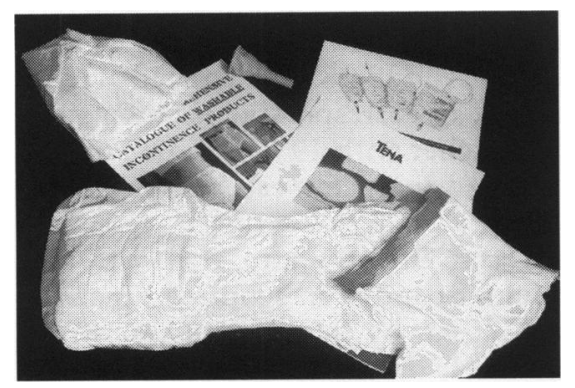

Figure 2 Pads and pants come in all shapes and sizes enabling tailoring to patient's needs. Some garments are disposable, others washable and other stitched into washable pants. For men with dribbling incontinence, penile pouches are available

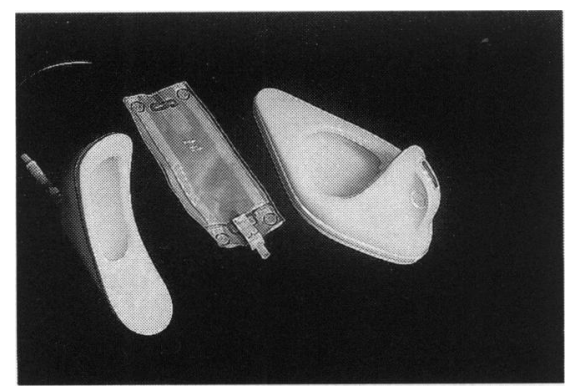

Figure 3 The Bridge urinal and the Bridge hand pan are designed for men and women to fit the body contour. They are for use in the sitting position, as in a wheelchair or on the side of the bed. The urinal may be attached to any drainage bag. The hand pan has a spout for easy emptying urethrography. Urodynamic studies, however, carry a risk of infection and they should only be carried out when the result is likely to affect the management.

\section{Treatments}

Apart from specific medical, surgical, physiological, and psychological treatments, many practical measures are helpful (see figures). These include keeping patients free from constipation, regular toileting regimens, and easy access to facilities. Hand rails may benefit mobility, reachable bottles or a bed-side commode with a night light may avoid falls. Velcro fasteners will aid dexterity. When pads are used, skin protection is essential and one-directional flow pads beneficial. Excessive evening drinks, alcohol, hypnotic drugs and diuretics after lunch-time should be avoided. Mattress covers and laundry services make the carers role less demoralising. Support and information are available from the Continence Foundation.

\section{Medical management}

The main pharmacological therapies focus on the treatment of detrusor instability. Anticholinergic agents are effective in reducing detrusor contractions but side-effects are common. Oxybutynin in low dosage minimises side effects and is well absorbed and its effectiveness has now been validated. ${ }^{10,11}$ It is proposed that oxybutynin and imipramine, which itself has sympathomimetic effects, a central action, and anti-cholinergic activity, are synergistic. Imipramine may cause symptomatic hypotension. Flavoxate continues to be used despite poor evidence of its efficacy. Whilst drugs may increase the bladder volume at which the unstable contractions occur, their use alone rarely leads to continence. They should be used in combination with habit training such as bladder drill, biofeedback, hypnotherapy or acupuncture.

Desmopressin reduces the nocturnal production of urine. ${ }^{12}$ Nocturnal polyuria is common in elderly men and may be due to reduced anti-diuretic hormone secretion, cardiac failure, low bladder capacity, or sleep distrubances. Desmopressin is used but leads to significant water overload and hyponatraemia.

Oestrogen replacement therapy in post-menopausal women reduces the incidence of urinary tract infections. There is no evidence that oestrogens alone are helpful in managing genuine stress incontinence but a combination of oestrogen and the alpha-adrenergic agonist, phenylpropanolamine is beneficial. ${ }^{13}$ Adjunctive oestrogen therapy may be helpful for the symptoms of urgency, frequency, and nocturia. Oestrogens are thought to raise the sensory threshold of the bladder. ${ }^{14}$

\section{Surgical management}

Surgery remains the most effective therapeutic intervention for women with genuine stress incontinence and males with detrusor instability secondary to outflow tract obstruction. For patients with severe detrusor instability and failed conservative treatments, surgery is the treatment of choice. The patients' physical frailty, mental state, and severity of symptoms influence decisions, but surgery need not be refused on grounds of age alone.

Surgery for genuine stress incontinence aims at elevating the bladder neck and proximal urethra into an intra-abdominal position, at supporting the bladder neck and, more recently, at increasing outflow resistance. The procedure is tailored to the patient. Artificial sphincters and collagen injections of the bladder neck under cystoscopic control are being evaluated.

The 'Clam' ileo-cystoplasty is the operation currently recommended for severe detrusor instability. ${ }^{15}$ In terms of continence the outcome is good, but may result in high residual volumes, requiring the use of intermittent selfcatheterisation. Copious mucus may be secreted by the ileal segment and there is the long-term risk of carcinomatous change. There is no role for the continued use of trans-trigonal phenol with its inherent risks, including fistulae.

In patients with detrusor instability secondary to outflow obstruction, surgery remains the therapeutic option of choice. Over half of the patients who undergo prostatectomy have an unstable bladder, although approximately two-thirds of these revert to a stable bladder within six months. Artificial urinary sphincters may be used in those who remain very wet as a consequence of prostatectomy.

\section{Physiotherapeutic, behavioural and other treatments}

Physiotherapy is well recognised in the management of stress incontinence, however increasing numbers of patients are being treated using a variety of 


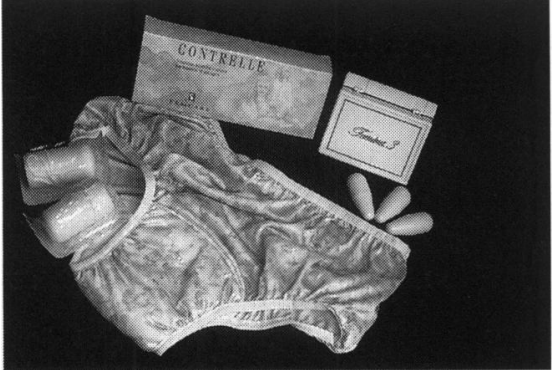

Figure 4 Weighted vaginal cones or the Contrelle disposable tampons are used in association with exercise to strengthen the pelvic floor muscles. Both are inserted vaginally and serve to support the bladder neck in stress incontinence. Garments (washable pants with inserted washable pads) are now available in attractive designs

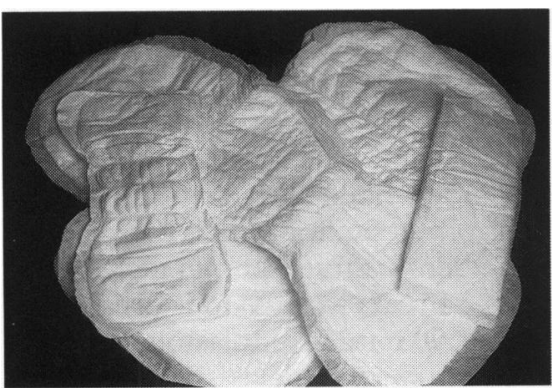

Figure 5 A wide variety of pads (and pants) is available and should be individualised, if necessary by pad weighing to avoid both leakage and excessively large pads

\section{Urinary incontinence and its management}

- incontinence of urine, although common, is not a natural sequel to growing old

- the majority of patients can be helped. Recovery has a dramatic effect on patient (and carer) morale

- an accurate diagnosis requires urodynamic studies

- drug treatments alone rarely lead to continence and should be combined with habit training

- surgical advances include injection of collagen into the bladder neck for genuine stress incontinence

- there is a need for a multidisciplinary approach to incontinence and more public education

techniques, with little consensus. ${ }^{16}$ Treatment modalities exist for ano-rectal incontinence, detrusor instability, and pelvic pain associated with incontinence.

Treatments generally target the pelvic floor muscles and involve muscle assessment, biofeedback, electrical stimulation, bladder training and the use of weighted vaginal cones. ${ }^{17}$ The combination of oestrogens with exercise is beneficial. Objective assessment with a perineometer, which allows visualisation of contractions on a computerised screen encouraging the patients to produce stronger contractions, may be used. Bladder training using electrical stimulation and relaxation therapy aim to suppress symptoms of urgency. Habit-training, to increase the voiding interval, hypnotherapy and acupuncture have all been used. The methods require the patients to be highly motivated and are time consuming, but can be used in elderly patients. ${ }^{18}$

Intermittent self-catheterisation has an important role in voiding disorders particularly those associated with neurological disease. The technique needs careful teaching and good medical support. Patients with longstanding severe incontinence and other problems such as a dense hemiplegia, may best be managed with long-term catheterisation, but this is a last resort.

Incontinence remains a stigma for the patients and places an onerous burden on the carer and it may affect acceptability for placement into residential care. Urinary incontinence may not always be curable but it is always possible to help improve the patients' quality of life. A Continence Awareness Campaign was proposed for March 1994 followed by a Ministerial Review for 1994/5 which should lead to further attention being directed towards this huge socio-medical problem.

1 Stanton SL. Gynaecological aspects. In: Mandlestram $\mathrm{D}$, ed. Incontinence and its management. London: Croom Helm, 1986; p 61 .

2 Bates P, Bradley WE, Glen E. Standardisation of terminology of lower urinary tract function. $f$ Urol 1979; 121: 551-4.

3 Brocklehurst JC. Urinary incontinence in the community - analysis of a MORI poll. $B M^{\mathcal{F}}$ 1993; 306: 852-4

4 Hodkinson E, McCafferty FG, Stott JN, Stou RW. Disability and dependency in elderly people in residential and hospital care. Age Ageing 1988; 17: 147-54.

5 Keane DP, Eckford SD, Shepherd AM, Abrams $P$. Referral patterns and diagnoses in women attending a urodynamic unit. $B M F$ 1992; 305: 308 .

6 Hilton P, Stanton SL. Algorithmic method for assessing urinary incontinence in elderly women. $B M F$ 1981; 282: 940-2.

7 Eastwood HDH, Warrell R. Urinary incontinence in the elderly female: prediction in diagnosis and outcome of management. $A g$ Ageing 1984; 13: 230-4.

8 Kong TK, Morris JA, Robinson JM, Brocklehurst JC. Predicting urodynamic dysfunction from clinical features in incontinent elderly women. Age Ageing 1990; 19: 257-63.

9 Barrett JA, Banks A, Roe B. Urodynamic investigations in elderly women. $B M \mathcal{F} 1992 ; 305$ 1438.

10 Cardozo LD, Coopr DJ, Versi E. Oxybutynin hydrochloride in the management of detruso instability. Neurol Urodynam 1987; 6: 256-7.
11 Moore $\mathrm{KH}$, Hay DM, Imrie AE, Watson A, Goldstein $M$. Oxybutynin ( $3 \mathrm{mg}$ ) in the treatment of women with idiopathic de

12 Knudsen HB, Rittig S, Pedersen JB, Norgaard JP, Djaakus JC. Long-term treatment of nocturnal enuresis with desmopression - influence on urinary output and haematological parameters. Neuro Urodynamics 1989; 8: 348-9.

13 Hilton P, Tweddell AL, Mayne C. Oral and intravaginal oestrogens alone and in combination with alpha-adrenergic stimulation in genuine stress incontinence. Int Urogynaecol $f$ 1990; 1: 80-6.

14 Fantl JA, Wyman JF, Anderson RL, Matt DW, Bunp RC. Post menopausal urinary incontinence: comparison between non-oestrogen supplemented and oestrogen supplemented supplemented and oestrogen supplem

15 Mundy AR, Stephenson TP. 'Clam' ileocystoplasty for the treatment of refractory urge inconplasty for the treatment of refractory urg

16 Mantle J, Eboo V. Physiotherapy for stres urinary incontinence: a national survey. $B M \mathcal{J}$ 1991; 302: 753-5.

7 Plevnik S. New methods for strengthening pelvic floor muscles. Proc 15th Annual Meeting International Continence Society. London: ICS 1985; pp 267-8.

18 Burgio KL, Whitehead WE, Engel BT. Urinary incontinence in the elderly. Bladder sphincte biofeedback and toilet skills training. Ann Intern Med 1985; 104: 507-15. 